\title{
Evaluation of proximal facial nerve conduction by transcranial magnetic stimulation
}

\author{
T N SCHRIEFER, * K R MILLS, $\dagger$ N M F MURRAY, C W HESS $\ddagger$ \\ From the Department of Clinical Neurophysiology, The National Hospital for Nervous Diseases, London, UK
}

SUMMARY A magnetic stimulator was used for direct transcutaneous stimulation of the intracranial portion of the facial nerve in 15 normal subjects and in patients with Bell's palsy, demyelinating neuropathy, traumatic facial palsy and pontine glioma. Compound muscle action potentials (CMAPs) thus elicited in the orbicularis oris muscle of controls were of similar amplitude but longer latency $(1.3 \mathrm{SD} 0.15 \mathrm{~ms})$ compared with CMAPs produced by conventional electrical stimulation at the stylomastoid foramen. No response to magnetic stimulation could be recorded from the affected side in 15 of 16 patients with Bell's palsy. Serial studies in two patients demonstrated that the facial nerve remained inexcitable by magnetic stimulation despite marked improvement in clinical function. In the patient with a pontine glioma, the CMAP elicited by transcranial magnetic stimulation was of low amplitude but normal latency. In six of seven patients with demyelinating neuropathy, the response to intracranial magnetic stimulation was significantly delayed. Magnetic stimulation produced no response in either patient with traumatic facial palsy. Although the precise site of facia nerve stimulation is uncertain, evidence points to the labyrinthine segment of the facial canal as the most likely location.

Transcutaneous electrical stimulation of the facial nerve at the stylomastoid foramen to elicit a compound muscle action potential (CMAP) was first described in 1952 by Botelho et al. ${ }^{1}$ Neurologists and otolaryngologists now routinely employ facial motor nerve conduction studies for documentation of the severity of facial nerve dysfunction and evaluation of prognosis for recovery in Bell's palsy. ${ }^{2} 3$ The technique is limited, however, because the nerve can normally be stimulated only in its extracranial course, distal to the site of pathology in Bell's palsy. Indirect techniques, such as the blink reflex, are necessary for the non-invasive study of the proximal segment of the facial nerve. ${ }^{4}$

Intracranial facial nerve stimulation is currently

Present addresses: *500 Cherry SE, Grand Rapids, Michigan, 49503, USA.

†Radcliffe Infirmary, Oxford OX2 6HE, UK.

$\ddagger$ Neurologische Universitatsklinik, Inselspital, CH 3010, Bern, Switzerland.

Address for reprint requests: $\operatorname{Dr} \mathrm{N}$ M F Murray, The National Hospital, Queen Square, London WCIN 3BG, UK

Received 16 April 1987 and in revised form 27 May 1987. Accepted I June 1987. employed intraoperatively to evaluate and preserve facial nerve function in patients with hemifaciab spasm, ${ }^{5}$ Bell's palsy, ${ }^{6}$ and cerebellopontine angle tumours. ${ }^{7}$ Such proximal facial nerve stimulation has, up to now, been limited to direct electrical stimulation of the exposed facial nerve in anaesthetised patients.

Recently percutaneous magnetic stimulation has been utilised to stimulate central motor pathways intracranially. ${ }^{8} \mathrm{~A}$ bank of capacitors is discharged through a coil placed over the patient's scalp, generating a strong, time-varying magnetic field. Magnetic fields, unlike electrical stimuli, pass readily through high resistance structures such as the scalp and skull. The magnetic field induces currents in the underlying neural tissue, with resultant excitation of neural structures.

Using this technique, reliable measurements of corticomotoneuronal conduction have been readily and painlessly obtained in controls and in a variety of pathological states..$^{9-11}$ We have applied this method to stimulate the facial nerve intracranially and assess conduction in its proximal segment. Results of this technique in controls and in patients with Bell's palsy, demyelinating neuropathy, traumatic facial weakness, and pontine glioma are reported. 
Patients and methods

\section{Patients}

Fifteen healthy controls, 16 patients with Bell's palsy, seven patients with demyelinating neuropathy, two patients with traumatic facial palsy, and one patient with a pontine glioma were studied. The procedure was performed bilaterally in all control subjects, 14 patients with Bell's palsy, four patients with demyelinating neuropathy, both patients with traumatic facial weakness, and the patient with a pontine glioma. The 15 control subjects included nine men and six women, age range 19-60 years with a mean of 32 years.

Bell's palsy patients included 10 men and six women, age range $17-56$ years, with a mean of 33 years. All Bell's palsy patients were referred specifically for this test. There was a typical history in all cases with no evidence of pre-existent neurological disease such as multiple sclerosis or neurosarcoidosis. The severity of the facial paralysis was not objectively graded. The onset of facial weakness occurred from 1 day to 3 years prior to testing. Serial evaluation was performed in two patients.

The seven patients with demyelinating neuropathy included four with hereditary motor and sensory neuropathy (HMSN) type 1, one with Guillain-Barré syndrome, one with chronic inflammatory demyelinating polyneuropathy, and one with a chronic acquired demyelinating polyneuropathy of uncertain type. Only one of these patients had symptoms or signs referable to the facial nerve, that being the Guillain-Barre patient who developed right facial weakness 3 weeks into his illness.

The two patients with traumatic facial nerve palsies suffered basilar skull fractures following falls which had occurred 6 months previously. Clinical and electromyographic evidence of complete loss of facial nerve function was found bilaterally in one patient and unilaterally in the other. The patient with the pontine glioma was evaluated as part of his pre-operative assessment.

Patients or control subjects with a history of epilepsy or previous neurosurgery, or with a cardiac pacemaker were excluded. The magnetic stimulator has been approved for clinical use by the National Hospital medical ethics committee.

\section{Methods}

Surface recordings were made with silver/silver chloride cup electrodes of $0.7 \mathrm{~cm}$ diameter. The recording electrode was taped over the motor region of the superior orbicularis oris muscle. ${ }^{12}$ The reference electrode was placed in the midline above the upper lip. If the latency of the subsequently recorded CMAP could not be measured with certainty, the position of the reference electrode was altered so as to produce a waveform with a sharp, initially negative deflection. Recording electrodes were maintained in the same position for electrical stimulation at the stylomastoid foramen and magnetic stimulation transcranially. Responses were amplified with a Medelec MS8 electromyograph machine (bandpass $20 \mathrm{~Hz}$ to $2 \mathrm{kHz}$ ) which was interfaced to a Research Machines $380 \mathrm{Z}$ microcomputer for waveform storage and subsequent analysis and plotting.

For extracranial electrical stimulation, a high voltage (maximum $750 \mathrm{~V}$ ) low output impedance stimulator (Digitimer D180) delivered stimuli to the facial nerve at the stylo- mastoid foramen. The stimulus was applied through bipolar saline soaked pad electrodes with an interelectrode distance of $2 \mathrm{~cm}$. The cathode was placed anterior to the mastoid process and the anode positioned so as to minimise stimulus artefact and inadvertent masseter stimulation. Two responses to supramaximal electrical stimuli were recorded. Latency was measured to the onset of the initial negative deflection. Amplitude was measured from the point of the initial negative deflection to the negative peak of the CMAP.

The magnetic stimulator used for transcranial facial nerve stimulation has been fully described previously. ${ }^{813} \mathrm{~A}$ flat circular copper coil (mean diameter $9 \mathrm{~cm}$ ), connected to a bank of capacitors was placed on the scalp. The optimal coil position for most patients was with the centre $3 \mathrm{~cm}$ posterior and $6 \mathrm{~cm}$ lateral to $\mathrm{Cz}(10-20$ system), so that the coil lay ipsilateral to the facial nerve being stimulated. However, this coil location was not rigidly adhered to. While stimulation was begun at this position in all patients, the coil was moved as necessary to produce an optimal response. As viewed from above, a clockwise inducing current proved preferable for right facial nerve stimulation and an anti-clockwise inducing current for left facial nerve stimulation.

Capacitors charged to a maximum of $3 \mathrm{kV}$ were discharged through the coil, generating a brief (peak at about $150 \mu \mathrm{s}$ ), intense magnetic field (peak of $2 \cdot 1$ Tesla at the centre of the coil at maximum output). This field passes unattenuated through the scalp and skull, inducing stimulating currents which are capable of exciting neural tissue. The intensity of the magnetic stimulus was gradually increased from subthreshold to supramaximal levels. Four responses to supramaximal stimuli were recorded. Latency and amplitude measurements were made as for electrical stimulation at the stylomastoid foramen. In Bell's palsy patients, the asymptomatic side was always studied first.

\section{Results}

\section{Normal subjects}

Table 1 summarises latency and amplitude measurements of CMAPs elicited from orbicularis oris in 15 healthy controls (30 sides) following electrical stimulation at the stylomastoid foramen and magnetic stimulation transcranially. Figure

Table 1 Data from 30 sides of 15 healthy subjects

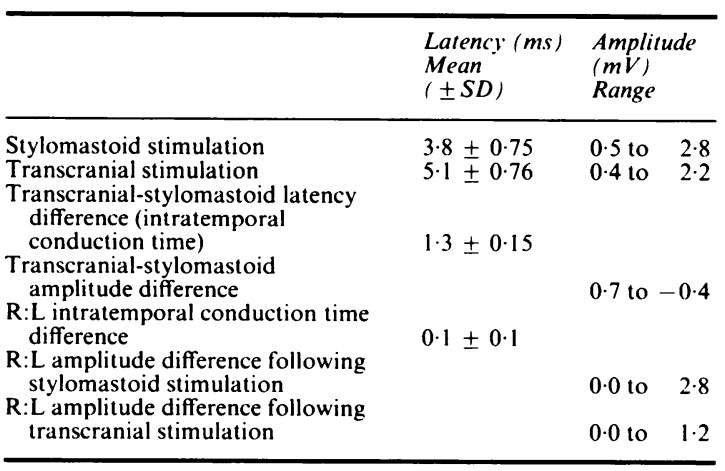


1 demonstrates a set of typical responses from a normal subject.

The orbicularis oris CMAP elicited by magnetic stimulation in normal subjects was of similar configuration and amplitude to that obtained by electrical stimulation at the stylomastoid foramen. The latencies obtained from the two stimulating sites were, however, quite dissimilar, with consistently longer latencies following magnetic stimulation. The mean latency difference between magnetic stimulation transcranially and electrical stimulation at the stylomastoid foramen was $1.3 \mathrm{SD} 0.15 \mathrm{~ms}$. This value represents the time necessary for the nerve action potential elicited magnetically in the proximal facial nerve to reach the point near the stylomastoid foramen where electrical stimulation occurs. Proximal facial nerve conduction time varied little between right and left sides in normal subjects. The mean side to side $(\mathbf{R}-\mathbf{L})$ latency difference in controls was $0 \cdot 1$ SD $0.1 \mathrm{~ms}$.

The CMAP elicited by magnetic stimulation was readily obtained at relatively low levels of stimulation. A supramaximal response was usually obtained with the magnetic field at only $50 \%$ of its maximal strength. This compares with a field strength of $70 \%$ maximum output routinely needed to record from hand muscles and $90 \%$ maximum output often required for the legs when the cortex is stimulated without facilitation.

The optimal site for facial nerve magnetic stimulation was fairly constant. Occasionally, supramaximal responses were obtained only after the coil had been moved $3-4 \mathrm{~cm}$ posterolaterally from its original position. In the majority of cases, however, the

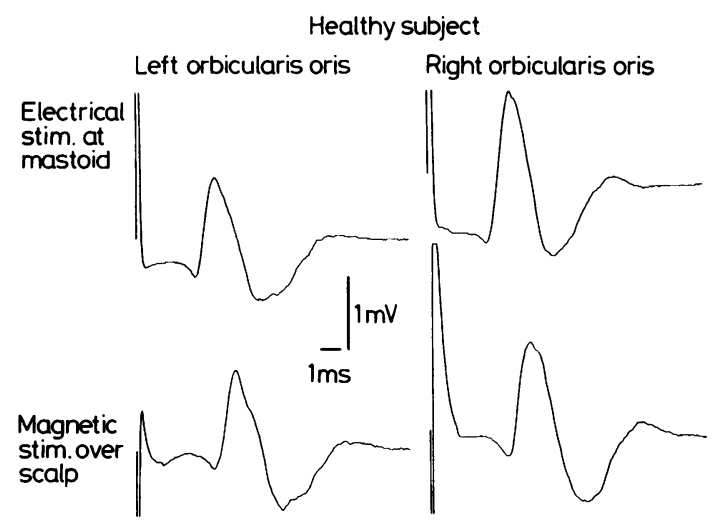

Fig 1 Compound muscle action potentials recorded over orbicularis oris on right and left sides of a healthy subject in response to electrical stimulation of the facial nerve at the ipsilateral stylomastoid foramen (upper traces) and magnetic stimulation over the scalp $6 \mathrm{~cm}$ lateral and $3 \mathrm{~cm}$ posterior to the vertex on the ipsilateral side (lower traces).

initial stimulation site proved adequate. A $1-2 \mathrm{~cm}$ movement in coil position typically produced no근 change in the amplitude or latency of the CMAP. The waveform of the CMAP elicited in orbicularis oris by transcranial magnetic stimulation was consistento from one supramaximal stimulation to the next. Once supramaximal stimulation was reached, furthero increases in stimulus intensity did not shorten theo latency or increase the amplitude-of the CMAP. Likewise, voluntary contraction of orbicularis oris during magnetic stimulation produced no changes in the

Table 2 Data from patients with Bell's palsy

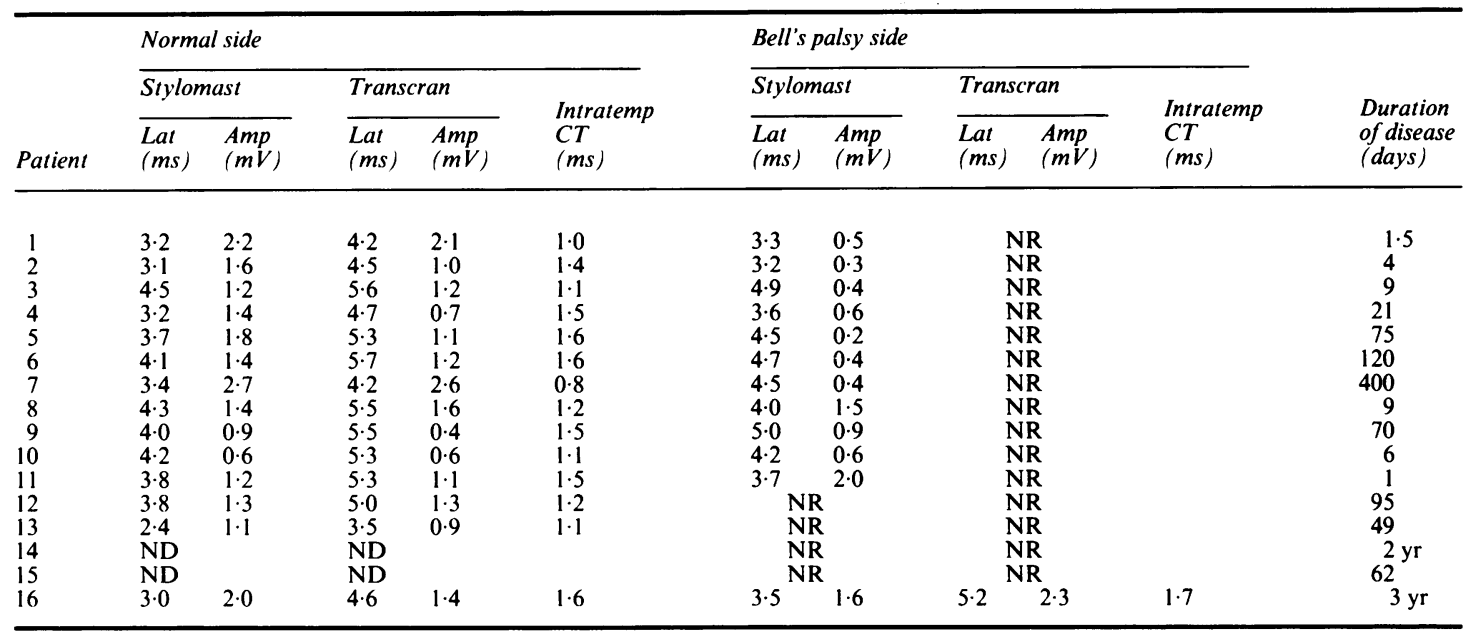

NR: no response; ND: not done; CT: conduction time. 
amplitude or waveform of the resultant CMAP. Responses at a latency consistent with excitation of the face area of the motor cortex were not seen, despite a thorough search with various coil positions, and with voluntary contraction of facial muscles.

\section{Patients}

Table 2 summarises the results of magnetic stimulation found in Bell's palsy patients. Findings in these 16 patients could be classified into four categories. The largest group consisted of seven patients with a low amplitude response in orbicularis oris to electrical stimulation of the facial nerve at the stylomastoid foramen with no CMAP recordable following magnetic stimulation transcranially. In these patients the onset of facial weakness preceded the examination by 2 days to 13 months (median 3 weeks). Four patients exhibited normal amplitude orbicularis oris CMAPs following electrical stimulation at the mastoid but no response to intracranial magnetic stimulation. Duration of facial palsy in these patients varied from 1 day to 11 weeks prior to testing. Figure 2 illustrates this finding in patient 11 (table 2) who developed Bell's palsy 1 day prior to testing. In four patients no response to electrical stimulation or magnetic stimulation was found. The onset of facial weakness in this subset of patients ranged from 7 to 12 weeks. In only one patient were both electrical and magnetic stimulation successful in eliciting a response on the affected side. In this case the orbicularis oris CMAP was of normal latency and amplitude. Clinically, this patient had noted the onset of facial weakness 3 years previously which subsequently improved without residual deficit.

Serial studies were performed in two patients. Patient 11 presented one day after developing an incomplete right facial palsy. Electrical stimulation of the right facial nerve at the stylomastoid foramen was of normal latency and amplitude. Magnetic stimulation transcranially produced no response (fig 2). On
Bell's Palsy patient

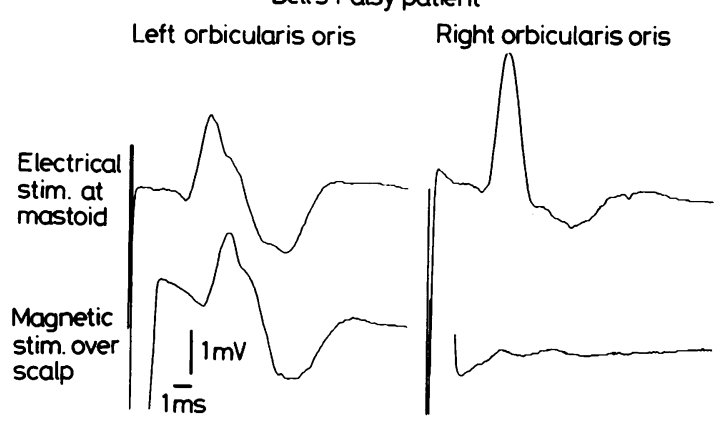

Fig 2 Compound muscle action potentials recorded over orbicularis oris in a patient with right sided Bell's palsy in response to electrical stimulation at the stylomastoid foramen (upper traces) and magnetic scalp stimulation (lower traces).

repeat examination 3 weeks later, eye closure had returned to normal and only a slight asymmetry of smile persisted. Conventional electrical stimulation of the right facial nerve remained normal. The facial nerve remained inexcitable, however, to transcranial magnetic stimulation.

Patient 3 (table 2) demonstrated similar findings. Nine days following the acute onset of right facial weakness, he was observed to have no clinically apparent facial nerve function on the right. Needle electromyography (EMG) revealed no active denervation and a single unit recruitment pattern. Electrical stimulation produced a low amplitude CMAP in orbicularis oris, but there was no response to magnetic stimulation. Two weeks later the facial weakness had almost completely resolved, the patient noticing only slight difficulty in playing the flute. The amplitude of the response to electrical stimulation had doubled. In spite of the improvement clinically and on conventional nerve conduction studies, mag-

Table 3 Data from patients with demyelinating neuropathy

\begin{tabular}{|c|c|c|c|c|c|c|c|}
\hline & & \multirow[b]{2}{*}{ Side } & \multicolumn{2}{|c|}{ Stylomastoid stimulation } & \multicolumn{2}{|c|}{ Transcranial stimulation } & \multirow{2}{*}{$\begin{array}{l}\text { Intratemp } \\
C T(m s)\end{array}$} \\
\hline \multicolumn{2}{|c|}{ Patient diagnosis } & & Lat (ms) & $A m p(m V)$ & $\overline{L a t}(m s)$ & $A m p(m V)$ & \\
\hline 1 & GBS & $\mathbf{R}$ & $3 \cdot 8$ & 0.5 & $7 \cdot 7$ & 0.2 & 3.9 \\
\hline 2 & HMSN I & $\mathbf{L}$ & $\begin{array}{l}3 \cdot 7 \\
6 \cdot 6\end{array}$ & $\begin{array}{l}1 \cdot 1 \\
0.5\end{array}$ & $\begin{array}{l}5 \cdot 2 \\
8 \cdot 2\end{array}$ & $\begin{array}{l}0.9 \\
0.6\end{array}$ & 1.5 \\
\hline 3 & HMSN I & $\mathbf{R}$ & $8 \cdot 4$ & $\begin{array}{l}0.5 \\
1.2\end{array}$ & $\begin{array}{r}0.2 \\
10.4\end{array}$ & $\begin{array}{l}0.0 \\
1.0\end{array}$ & $\begin{array}{l}1.6 \\
2.0\end{array}$ \\
\hline 4 & HMSN I & $\mathbf{R}$ & $5 \cdot 4$ & 0.3 & $7 \cdot 2$ & 0.3 & 1.8 \\
\hline & & $\mathbf{L}$ & $5 \cdot 5$ & 0.6 & $7 \cdot 8$ & 0.5 & $2 \cdot 3$ \\
\hline 5 & HMSN I & $\mathbf{R}$ & $6 \cdot 0$ & $1 \cdot 5$ & $7 \cdot 8$ & 0.2 & 1.8 \\
\hline & & L & 6.5 & 0.7 & $8 \cdot 2$ & 0.4 & 1.7 \\
\hline $\begin{array}{l}6 \\
7\end{array}$ & CPIP & $\begin{array}{l}\mathbf{R} \\
\mathbf{R}\end{array}$ & $16 \cdot 7$ & 0.8 & NR & & \\
\hline I & DPN & $\begin{array}{l}\mathbf{R} \\
\mathbf{L}\end{array}$ & $\begin{array}{l}4 \cdot 8 \\
4 \cdot 2\end{array}$ & $\begin{array}{l}2 \cdot 7 \\
2 \cdot 5\end{array}$ & $\begin{array}{l}6.0 \\
6.0\end{array}$ & $\begin{array}{l}2.5 \\
2.5\end{array}$ & $\begin{array}{l}1 \cdot 2 \\
1 \cdot 8\end{array}$ \\
\hline
\end{tabular}

NR: no response; GBS: Guillain-Barré syndrome; HMSN I: hereditary motor and sensory neuropathy type I; CPIP: chronic progressive inflammatory polyneuropathy; DPN: demyelinating peripheral neuropathy of unknown cause. 
netic stimulation of the facial nerve transcranially produced no response.

Taking the group of Bell's palsy patients as a whole, in only one of 16 patients studied was intracranial magnetic stimulation successful in producing a response on the affected side. In 11 of 16 cases, a response to electrical stimulation at the stylomastoid foramen could be recorded in the absence of a response to magnetic stimulation intracranially. In no case, however, was the converse true.

Results from patients with demyelinating neuropathies are outlined in table 3 . Of this group, only patient 1 complained of symptoms referable to the facial nerve. He noted the acute onset of right facial weakness and numbness 3 weeks after numbness and ascending weakness in his arms and legs had been diagnosed as Guillain-Barré syndrome. In contrast to patients with Bell's palsy, responses to magnetic stimuli could be elicited in all but one subject. Of the seven patients studied six had prolonged proximal conduction times on at least one side. Five of these six patients had no symptoms of facial nerve dysfunction.

In the two patients with traumatic facial nerve palsies, neither electrical stimulation at the stylomastoid foramen nor magnetic stimulation intracranially elicited a recordable response in orbicularis oris. Masseter contraction was observed following magnetic stimulation in both patients.

The absence of a response to transcranial magnetic stimulation in acute Bell's palsy proved useful in interpreting the results of the patient with a pontine glioma. Four months prior to evaluation, this 18 year old man developed difficulty closing his left eye, trouble whistling, and impaired taste on the left side of the tongue. Bell's palsy was diagnosed. After several days taste returned to normal and his facial weakness was slowly improving. One month later, however, he noted horizontal diplopia on left lateral gaze. At the time of the examination the patient had bilateral papilloedema and incomplete palsies of the left abducens and facial nerves. A MRI scan revealed a pontomedullary mass consistent with a brainstem glioma. The patient was referred for magnetic stimulation to confirm the clinical impression that his facial weakness was secondary to the intramedullary tumour and not Bell's palsy.

The results are illustrated in fig 3 . The response to left facial nerve stimulation at the stylomastoid foramen is of low amplitude compared with the right side. Transcranial magnetic stimulation elicited a CMAP of similar waveform and amplitude with an appropriately longer latency. The preserved response to magnetic stimulation in this patient contrasts with our findings in Bell's palsy (fig 2), where typically no proximal response can be elicited. The assessment

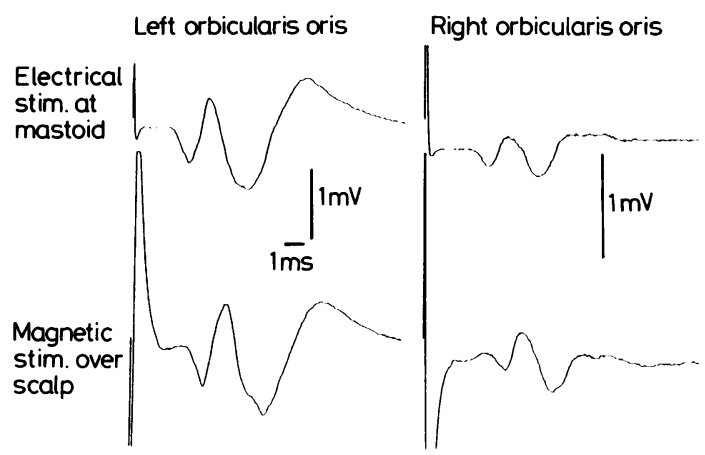

Fig 3 Compound muscle action potentials recorded over orbicularis oris in a patient with a pontine glioma in whom it was unclear whether the right facial weakness was due to an intrinsic or peripheral facial nerve lesion. Electrical stimulation at the stylomastoid foramen (upper traces) and magnetic stimulation over the scaip (lower traces) gave responses of similar amplitude and normal latency difference on the affected side, suggesting the lesion was central.

from transcranial magnetic stimulation was that the patient's facial palsy was secondary to his intramedullary tumour and not Bell's palsy. At surgery the glioma involved the left abducens nucleus an motor fibres of the facial nerve as they looped aroun $\$$ the abducens nucleus.

\section{Discussion}

This study demonstrates that magnetic stimulatio can be used to evaluate proximal facial nerve condue tion directly and noninvasively. The facial nerve is excited transcranially by magnetic stimuli, and the resultant orbicularis oris CMAP is recorded. Conventional electrical stimulation of the facial nerve at the stylomastoid foramen produces a CMAP of similar amplitude and configuration, but shorter latency. If the latency of the electrical response is subtracted $\overrightarrow{\vec{O}}$ from the latency of the magnetic response, a measurement of proximal facial nerve conduction is obtained.

The exact site of facial nerve excitation during magnetic stimulation is difficult to determine. Uncertainty over the precise site of stimulation has been noted $:$ with magnetic stimulation of both central and periph- 3 . eral neural structures. ${ }^{13}{ }^{14}$ However, reasonable con- $\delta$ clusions can be drawn from the available data.

To begin with, it seems certain that the response to 을 magnetic stimulation is due to lower motor neuron rather than supranuclear excitation. Magnetic stimulation of central motor tracts is characterised by variability of CMAP waveforms with successive identical of stimuli and facilitation of the response with voluntary $N$ contraction. ${ }^{13}$ Neither of these features was present $\underset{\omega}{\mathcal{E}}$ 
with magnetic facial stimulation. The mean CMAP amplitude elicited by intracranial magnetic facial nerve stimulation $(1.4 \mathrm{SD} 0.5 \mathrm{mV})$ was only $10 \%$ less than that elicited by peripheral electrical stimulation $(1.6 \mathrm{SD} 0.6 \mathrm{mV})$. This decrease in amplitude is much less than has been previously noted when central motor tracts have been stimulated magnetically. ${ }^{10}$ As will be discussed below, the mean latency of $5.1 \mathrm{~ms}$ following magnetic stimulation to the onset of the orbicularis oris CMAP is best explained by a peripheral stimulation site.

Our measurements of proximal facial nerve conduction following magnetic stimulation are comparable with results of intraoperative electrical facial nerve stimulation. Møller and Jannetta stimulated the facial nerve at the root entry zone with a needle electrode in seven patients with hemifacial spasm and found a mean latency of $4.65 \mathrm{~ms}$ to orbicularis oculi. ${ }^{5}$ Again using needle stimulation of the facial nerve at the root entry zone, the same authors reported a mean latency of $4.9 \mathrm{~ms}$ to the orbicularis oculi and $5.3 \mathrm{~ms}$ to mentalis in four patients with hemifacial spasm. ${ }^{15}$ These data are consistent with our finding of a mean latency of $5.1 \mathrm{~ms}$ to orbicularis oris following magnetic stimulation, and suggest that magnetic stimulation excites the facial nerve directly in its proximal course.

Attempts to localise the precise facial nerve stimulation site by inference from latency determinations prove difficult. A latency of only $0.3 \mathrm{~ms}$ separates the facial nerve root entry zone from its entry through the porus acousticus. ${ }^{16}$ In view of the variation in latencies to orbicularis oris in healthy controls following magnetic stimulation, one cannot even be sure that the facial nerve stimulation site is identical in all subjects. With this in mind, a cautious interpretation is appropriate. The most that can be concluded from latency data alone is that magnetic stimulation of the facial nerve occurs between the root entry zone and the beginning of the facial canal.

We found the mean latency between facial nerve magnetic stimulation transcranially and electrical stimulation near the stylomastoid foramen to be $1.3 \mathrm{~ms}$. Knowing the length of the facial nerve to be approximately $60 \mathrm{~mm}$ from the root entry zone to the stylomastoid foramen, ${ }^{17}$ a proximal facial nerve conduction velocity of $46 \mathrm{~m} / \mathrm{s}$ can be calculated. If, however, the facial nerve is actually stimulated at its entrance into the facial canal, a conduction velocity of $26 \mathrm{~m} / \mathrm{s}$ over the $30 \mathrm{~mm}$ segment to the stylomastoid foramen can be calculated. Both of these figures are consistent with previously reported values for facial nerve conduction. ${ }^{2} 71618$

When complete facial nerve transection has occurred during surgery for removal of a cerebellopontine angle tumour, facial twitches could still be elicited postoperatively on the denervated side with magnetic stimulation (HI Sabin and L Symon, personal communication). For this to occur, the site of magnetic stimulation must have been distal to the point of facial nerve transection, that is, at or distal to the internal auditory meatus.

The results of this technique's application to Bell's palsy patients are of considerable interest. Although 11 patients with recent Bell's palsy had orbicularis oris CMAPs elicited by electrical stimulation near the stylomastoid foramen, none demonstrated a response to magnetic stimulation of the facial nerve. This implies that the site of magnetic stimulation must be at or proximal to the lesion in Bell's palsy. Pathological and physiological data have localised this lesion to the facial nerve as it enters the labyrinthine segment of the facial canal, ${ }^{23}$ in keeping with our estimation of the excitation site in normal subjects.

The findings in patient 11 illustrate both the potential applications and limitations of magnetic stimulation for the evaluation of facial weakness. One day following the onset of partial facial weakness, the orbicularis oris CMAP elicited by conventional electrical stimulation near the stylomastoid foramen was of normal latency and amplitude. In contrast, no response could be elicited by magnetic transcranial stimulation. This clearly demonstrated a peripheral facial nerve lesion, and confirmed the clinical diagnosis of Bell's palsy. Inability to activate patient 11's facial nerve magnetically persisted 3 weeks later, even as clinical function returned to near normal and conventional facial nerve conduction studies remained unaffected. The only other patient to have follow up studies, patient 3 , also failed to generate a response to magnetic stimulation on repeat examination despite marked clinical improvement. Thus, absence of a response following magnetic stimulation of the proximal facial nerve does not necessarily portend a poor prognosis in Bell's palsy. Likewise magnetic stimulation, as described here, does not reliably predict prognosis for recovery of facial nerve function in Bell's palsy.

The absence of a response to proximal facial nerve stimulation in Bell's palsy patients is most likely because the stimulus is applied to the area of demyelination, that is, the labyrinthine segment of the facial canal. The stimulation threshold of segmentally demyelinated nerve is higher than that of surrounding, normal nerve. ${ }^{19}$ One may hypothesise that if the site of magnetic stimulation could be moved proximally or distally to the site of demyelination in Bell's palsy, these segments would remain magnetically excitable. Advances in magnetic stimulator and, particularly, coil design may provide the means to stimulate these segments and thus permit measurement of the degree of conduction block.

Magnetic stimulation adds little to conventional 
66

nerve conduction studies in the evaluation of chronic, complete facial nerve palsies. Not surprisingly, when no facial nerve response can be elicited distally with electric stimulation, no response can be elicited proximally by magnetic stimulation.

Prolonged intracranial facial nerve conduction times were found in six of seven patients with demyelinating neuropathies. Assuming a $30 \mathrm{~mm}$ distance from the entry of the facial nerve canal to the stylomastoid foramen, ${ }^{17}$ conduction velocities of 8 to 15 $\mathrm{m} / \mathrm{s}$ can be calculated over the intratemporal segment of these abnormal facial nerves. This is consistent with the finding of prolonged facial motor distal latencies in patients with demyelinating neuropathies. ${ }^{20}$

In conclusion, magnetic stimulation of the intracranial facial nerve, probably in its labyrinthine segment, may well prove to be a useful adjunct to electrical stimulation at the stylomastoid foramen for evaluating acute and/or traumatic facial nerve palsy. Initial expectations that the method would allow quantification of conduction block in Bell's palsy have not been realised with currently available coil designs. However, facial palsy due to an intrinsic brainstem lesion has been differentiated from paralysis due a lesion in the intratemporal course of the nerve. Furthermore, documentation of proximal slowing of facial nerve conduction may be useful in the evaluation of demyelinating peripheral neuropathy, especially early Guillain-Barré syndrome, before more general slowing of peripheral nerve conduction occurs.

We thank the physicians of The National Hospital for permission to study their patients and Dr AT Baker, Mr R Jalinous and Dr IL Freeston who devised and constructed the magnetic stimulator. This work was supported by grants from The Multiple Sclerosis Society (KRM and NMFM), The Rotary Foundation of Rotary International (TNS) and The Roche Research Foundation (CWH).

\section{References}

1 Botelho SY, Deaterly CF, Comroe JH. Electromyogram from orbicularis oculi in normal persons and in patients with myasthenia gravis. Arch Neurol Psychiatry 1952;67:348-53.

2 Esslen E. The Acute Facial Palsies. Berlin: SpringerVerlag, 1977.

3 Silverstein H, McDaniel AB, Hyman SM. Evoked serial electromyography in the evaluation of the paralyzed
Schriefer, Mills, Murray, Hess

face. Am J Otol 1985;(Suppl 6):80-7.

4 Kimura J, Giron LT, Young SM. Electrophysiological study of Bell's palsy: electrically elicited blink reflex in assessment of prognosis. Arch Otolaryngol 1976;102:140-3.

5 Møller AR, Jannetta PJ. On the origin of synkinesis in hemifacial spasm: results of intracranial recordings. J Neurosurg 1984;61:569-76.

6 Gantz BJ, Gmür A, Fisch U. Intraoperative evoked electromyography in Bell's palsy. Am J Otolaryngol 1982;3:273-8.

7 Richmond IL, Mahla M. Use of antidromic recording to monitor facial nerve function intraoperatively. Neurology 1985;16:458-62.

8 Barker AT, Jalinous R, Freeston IL. Non-invasive magnetic stimulation of human motor cortex. Lancet 1986;i:1106-7.

9 Barker AT, Freeston IL, Jalinous R, Jarratt JA. Clinical evaluation of conduction time measurements in central motor pathways using magnetic stimulation of the human brain. Lancet 1986;i:1325-6.

10 Hess CW, Mills KR, Murray NMF. Measurement of central motor conduction in multiple sclerosis by magnetic brain stimulation. Lancet 1986;ii:355-8.

11 Mills KR, Murray NMF, Hess CW. Magnetic and electrical transcranial brain stimulation: physiological mechanisms and clinical applications. Neurosurgery 1987;20:164-8.

12 Walthard KM, Tchicaloff M. Motor points. In: Licht S ed. Electrodiagnosis and Electromyography. Newo Haven: Elizabeth Licht, 1961:153-70.

13 Hess CW, Mills KR, Murray NMF. Responses in smal hand muscles from magnetic stimulation of the humar brain. J Physiol (Lond) 1987;388:397-419.

14 Smith SJM, Murray NMF. Electrical and magnetic stimulation of lower-limb nerves and roots. Muscl Nerve 1986;9:652-3.

15 Møller AR, Jannetta PJ. Blink reflex in patients with hemifacial spasm: observations during microvascular decompression operations. J Neurol Sci 1986;72: $171-82$.

16 Møller AR, Jannetta PJ. Hemifacial spasm: results of electrophysiologic recording during microvascular decompression operations. Neurology 1985;35:969-74.

17 Diamond CW. Anatomy and physiology. In: Diamond C, Frew I, eds. The Facial Nerve. Oxford University Press, 1979:1-36.

18 Zander Olsen P. Prediction of recovery in Bell's palsy. Acta Neurol Scand 1975;(Suppl 61):1-121.

19 Bostock H, Sears TA, Sherratt RM. The spatial distribution of excitability and membrane current in normal and demyelinated mammalian nerve fibres. $J$ Physiol (Lond) 1983;341:41-58.

20 Dyck PJ, Lambert EH, Mulder DW. Charcot-MarieTooth disease: nerve conduction and clinical studies of a large kinship. Neurology 1963;13:1-11. 